\title{
Theory of inventive problem solving (TRIZ): Case study
}

\section{Maida Bárbara Reyes Rodríguez ${ }^{1}$, Jorge Laureano Moya Rodríguez ${ }^{1}$, Jandecy Cabral Leite ${ }^{1}$, Ángel Silvio Machado Rodríguez ${ }^{2}$}

\author{
${ }^{1}$ Instituto de Tecnologia E Educação Galileo Da Amazônia (ITEGAM). Av.Joaquim Nabuco No 1950. Centro, Manaus-AM. \\ CEP:69005-080. Tel: +55 9235846145. \\ ${ }^{2}$ Universidad Central "Martha Abreu” de Las Villas (UCLV). Carretera a Camajuaní 5,5 Km. Santa Clara. Villa Clara. Cuba. \\ Email: maidabrr@gmail.com,jorgemoyar@gmail.com,jandecy.cabral@itegam.org.br,angelmr@uclv.edu.cu.
}

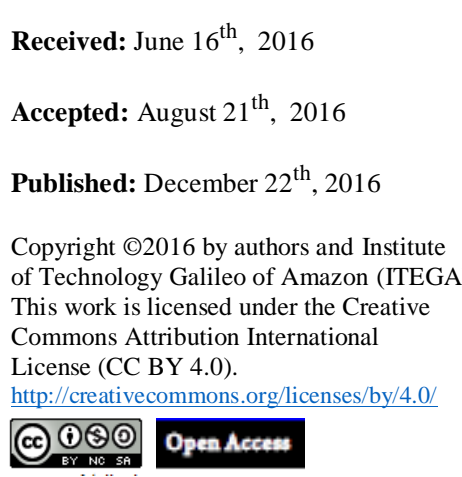

\begin{abstract}
TRIZ comes from the Russian acronym of the phrase Theory of Inventive Problem Solving ("Tieoriya Riesheniya Izobrietatielskij Zadach" or Теория решения изобретательских задач). This theory for solving problems and projects invention was developed by the Russian inventor Genrich Altshuller and his colleagues since 1946. This methodology is based on the belief that there are universal principles of the invention, which are the basis for creative innovations that assist in the advancement of technology. Depending on the amount of data and the availability of data analysis methods, problems can be classified . as structured, semi-structured or unstructured. Structured Problems are those whose solution depends on a single series of steps. The data set is known and a known sequence, a structured data identical problem will always have the same solution must be followed. In unstructured problems there is not an algorithm that ensures to reach an optimal solution, either because there is insufficient information on the factors that affect the solution or because there are many potential factors, which can not be formulated with any algorithm that guarantees a unique solution is correct. Actually, TRIZ improves the methodology for problem solving structured by applying its principles to the early stages of conventional structured methodology more creative and advanced measures that make the resolution process more efficient and effective, also this methodology can be applied to unstructured problems. In this paper, an analysis of the methods of TRIZ Theory is made and two case studies are addressed through this methodology.
\end{abstract}

Keywords: TRIZ, Problem.

\section{Teoría de solución de los problemas de inventiva (TRIZ): Estudio de casos}

\begin{abstract}
RESUMO
TRIZ proviene de las siglas en ruso de la frase Teoría para Resolver Problemas de Inventiva ("Tieoriya Riesheniya Izobrietatielskij Zadach" о Теория решения изобретательских задач). Esta teoría para la solución de problemas y proyectos de invención, fue desarrollada por el inventor ruso Genrich Altshuller y sus colegas desde 1946. Esta metodología se basa en la creencia de que hay principios universales de la invención que son la base para las innovaciones creativas que ayudan en el avance de la tecnología. Dependiendo de la cantidad de datos y la disponibilidad de los métodos de análisis de datos, los problemas pueden clasificarse como estructurados, semiestructurados o no estructurados. Los Problemas estructurados son aquellos cuya solución depende de una sola serie de pasos. El conjunto de datos es conocido y debe seguirse una secuencia conocida, un problema estructurado con datos idénticos siempre tendrá la misma solución. En los problemas no estructurados no hay un algoritmo que permita llegar a una solución óptima, ya sea porque no hay información suficiente sobre los factores que afectan la solución o porque hay tantos factores potenciales, que no puede formularse ningún algoritmo que garantice una solución única que sea correcta.
\end{abstract}

Palabras Claves: Manual, Gestão de Qualidade, Padronização, E-manual, E-comunicação.

\section{INTRODUCCIÓN}

Después de la Segunda Guerra Mundial, la alta calidad, y los productos tecnológicos avanzados de los Estados Unidos dominaron los mercados mundiales. Con la crisis del petróleo de los años 70, sin embargo, muchas de las ventajas económicas asociadas a los precios baratos del petróleo se perdieron y las economías recuperadas de Europa y de Asia emergieron como fuertes competidores en muchas áreas de producción. Las tecnologías 
innovadoras de los E.E.U.U. ya no podían aislar más a las industrias de los productores europeos y asiáticos. Los años 90 han vieron la recuperación de muchas industrias de los E.E.U.U., especialmente la industria automotriz. Esto ha sido debido en parte a la influencia de muchas metodologías japonesas de calidad introducidas, por ejemplo las del Dr. Kaoru Ishikawa [1], del Dr. Masao Kogure [2], del Dr. Yoji Akao [3], del Dr. Noriaki Kano [4], de Masaaki Imai [5], y muchos otros. Estos métodos de la calidad han ayudado a industrias de los E.E.U.U. a reducir defectos, mejorar la calidad, abaratar los costos, y han estado más enfocados hacia el consumidor. Mientras que la brecha de la calidad con los países como Japón se ha hecho más pequeña.

Mientras que hay muchas metodologías de diseño y desarrollo ampliamente usadas tales como Quality Function Deployment [6], las mismas muestran qué solucionar pero no siempre cómo solucionar los embotellamientos de la tecnología que se presentan . Otra técnica denominada Reviewed Dendrogram [7], se basa en la experiencia de los diseñadores la cual puede estar limitada a ciertas áreas de experiencia. Así una solución que pudiera ser más simple y barata puede no ser tenida en cuenta debido a la falta de experiencia en esa área de los diseñadores. Si la solución está fuera del campo de la experiencia del Ingeniero, entonces ¿como podría él imaginar tal solución? Usando la TRIZ, el ingeniero puede ser capaz de explorar soluciones de diseño aún en campos de actuación que no sea el suyo propio.

\section{MATERIALES Y MÉTODOS II.1 ¿QUÉ ES TRIZ?}

TRIZ es una metodología propuesta por Genrich Altshuller para resolver problemas de inventiva. Durante más de 60 años de desarrollo de esta teoría se han visto involucrados en la misma cientos de científicos e inventores. Se ha utilizado para desarrollar la misma la experiencia práctica de miles de estos científicos e inventores así como hombres de negocios, ingenieros, managers, etc. Fueron consultadas más de 3 millones de patentes a nivel mundial y ha sido aplicada en innumerables problemas de Ingeniería y también en los negocios, la administración, la gestión y las ciencias sociales [8].

\section{II.2 BREVE HISTORIA DE LA TRIZ}

A continuación se exponen de manera cronológica los principales hitos de la historia de la TRIZ [9].

- 1926 - Nace Genrich Altshuller

- 1946 - Comienzo de la TRIZ

- 1956 - Primer artículo de Altshuller sobre TRIZ : "Psychology of Innovation"

- 1959 - Primer Algoritmo para la Solución de Problemas de Inventiva (ARIZ)

- 1960 - Artículo de Altshuller titulado "Como se realizan los descubrimientos", el cual constituye el inicio de la aplicación de la TRIZ a la ciencia.

- 1961 - Primer libro sobre TRIZ de Altshuller : "Aprendiendo a Inventar"
- 1964 - Segundo libro de Altshuller: "Bases del proceso de Invención"

- 1969 - Libro de Altshuller: "Algoritmo de Invención”

\section{- Primer Centro Educacional de Bakú}

- Primer Laboratorio para investigaciones sobre creatividad

- Primer seminario sobre TRIZ.

- Comienzo del establecimiento de escuelas de

TRIZ en diferentes lugares de la antigua URSS.

- 1971 - Surgimiento de ARIZ - 71, que incluye los 40 Principios de Innovación, la Tabla de Contradicciones y materiales acerca de la evolución de sistemas tecnológicos.

- 1979 - Libro de Altshuller "La creatividad como una ciencia exacta",

- 1980 - Primer Congreso de desarrolladores de TRIZ en Petrozavodsk

- 1985 - Altshuller presenta la Estrategia de Vida para la Creatividad Individual

- Primera presentación del "Método para Resolver Problemas Científicos" (por B. Zlotin y A. Zusman).

- Primera presentación del "Método para Resolver Problemas de la vida real" (por B. Zlotin y A. Zusman).

- Primera Compañía privada TRIZ Engineering Progress, establecida en Kishiniev

- 1986 - Se establece la TRIZ Association

- 1998 - Muerte de Altshuller

\section{II.3 TRABAJO REALIZADO POR EL CREADOR DE LA TRIZ PARA DESARROLLAR ESTA TEORÍA.}

Altshuller tamizó 1.500.000 patentes, quedándose con 200.000 de ellas tratando de buscar solo los problemas inventivos y la forma en que fueron resueltos. De estas solo 40.000 patentes fueron consideradas por inventivas en el año 1990. A la fecha se estiman en más de dos millones las patentes tamizadas por TRIZ. Siguen siendo solo unas 45000 las patentes inventivas (que incluyen un nuevo fenómeno) y el resto son solo mejoras rutinarias o nuevos conceptos. Altshuller categorizó estas patentes de una manera nueva.

En vez de clasificarlas por industria, tal como automotriz, aeroespacial, etc., él quitó el tema para descubrir el proceso de la solución de problemas. Encontró que los mismos problemas habían sido solucionados a menudo una y otra vez usando uno de solamente cuarenta principios inventivos fundamentales. Si los últimos inventores hubieran tenido conocimiento del trabajo de los primeros, las soluciones se habrían. 
Tabla 1: Resultados de la pesquisa de Altshuller.

\begin{tabular}{|l|l|l|c|}
\hline Nivel & Grado de inventiva & Requerimientos de conocimientos & $\begin{array}{l}\% \\
\text { Solución }\end{array}$ \\
\hline 1 & $\begin{array}{l}\text { Soluciones aparentes. Una mejora sencilla de un sistema } \\
\text { técnico. }\end{array}$ & $\begin{array}{l}\text { Conocimiento disponible dentro de un área de } \\
\text { producción/ aplicación relevante del sistema. }\end{array}$ & $32 \%$ \\
\hline 2 & $\begin{array}{l}\text { Mejoras menores. Una invención que incluye la resolución } \\
\text { de una contradicción técnica }\end{array}$ & $\begin{array}{l}\text { Conocimiento de áreas diferentes dentro de un área } \\
\text { relevante del sistema. }\end{array}$ & 45\% \\
\hline 3 & $\begin{array}{l}\text { Mejoras mayores. Una invención que contiene una } \\
\text { resolución de una contradicción física. }\end{array}$ & $\begin{array}{l}\text { Conocimiento de otras áreas de producción/ aplicación. } \\
\text { que contiene una solución de "ruptura". }\end{array}$ & $18 \%$ \\
\hline 4 & $\begin{array}{l}\text { Descubrimiento de nuevos fenómenos y substancias. } \\
4 \%\end{array}$ & Todo lo que es conocible & $1 \%$ \\
\hline
\end{tabular}

Fuente: Adaptado de [10].

Además de la TRIZ, han surgido a través de los años otras muchas teorías para la solución de problemas que se usan mucho en la actualidad, entre las que se encuentran:

\section{Década de los 50s:}

Brainstorming [11].

Morphological Analysis [12].

Value Engineering Analysis [13][14].

Taguchi Methods [15].

Fishbone Diagram [16].

\section{Década de los 60s:}

Synetics [17].

Revealing and Resolving Contradictions [18]

Control of though in creative work [19][20].

Systemic Design [21][22].

Technological Forecasting [23].

\section{Década de los 70s:}

Innovative Design [24].

Evolution of Technology [25].

Quality Function Deployement [26].

Psychological activation [27].

\section{Década de los 80s:}

Third Wave [28].

Six Sigma [29].

HAZOP and FMEA [30].

DFMA [31].

\section{Década de los 90s:}

Concurrent Engineering [32].

TOC [33].

Reengineering [34].

\section{II.4. APLICACIONES DE LA TRIZ.}

Esta teoría puede y ha sido aplicada en:

- Resolver problemas

- Mejorar productos

- Rediseñar operaciones

- Comprender las personas

\section{II.5. ESTRUCTURA DE LA TRIZ.}

La TRIZ está estructurada por las siguientes partes:

- Matriz de Contradicciones, que contiene 39 parámetros de Ingeniería y 40 Principios de Inventiva.

- Análisis Sustancia - Campo. (76 soluciones estándares)

- ARIZ (Algoritmo de Resolución de Problemas Inventivos)

- $\quad$ AFD (Determinación de fallas anticipadas)

Normalmente lo más usado en solución de problemas de Ingeniería es la Matriz de contradicciones.

\section{II.5.1 PARÁMETROS DE INGENIERÍA A SER MEJORADOS.}

Los 39 parámetros de Ingeniería que componen la matriz de contradicciones de Altshuller que pueden y deben ser mejorados aparecen en la tabla 2. 
Tabla 2. Parámetros de Ingeniería a ser mejorados

\begin{tabular}{|ll|}
\hline 1. & Peso del objeto móvil \\
\hline 2. & Peso del objeto estacionario \\
\hline 3. & Longitud del objeto móvil \\
\hline 4. & Longitud del objeto estacionario \\
\hline 5. & Área del objeto móvil \\
\hline 6. & Área del objeto estacionario \\
\hline 7. & Volumen del objeto móvil \\
\hline 8. & Volumen del objeto estacionario \\
\hline 9. & Velocidad \\
\hline 10. & Fuerza \\
\hline 11. & Tensión/Presión \\
\hline 12. & Forma \\
\hline 13. & Estabilidad de la composición \\
\hline 14. & Resistencia \\
\hline 15. & Tiempo de acción del objeto en movimiento \\
\hline 16. & Tiempo de acción del objeto estacionario \\
\hline 17. & Temperatura \\
\hline 18. & Brillantez \\
\hline 19. & Energía consumida por el objeto en movimiento \\
\hline 20. & Energía consumida por el objeto estacionario \\
\hline 21. & Potencia \\
\hline 22. & Pérdida de energía \\
\hline 23. & Pérdida de substancia \\
\hline 24. & Pérdida de información \\
\hline 25. & Pérdida de tiempo \\
\hline 26. & Cantidad de substancia \\
\hline 27. & Confiabilidad \\
\hline 28. & Precisión de la medición \\
\hline 29. & Precisión de manufactura \\
\hline 30. & Factores dañinos que actúan sobre el objeto desde el \\
\hline exterior & \\
\hline 31. & Factores dañinos provocados por el objeto \\
\hline 32. & Manufacturabilidad \\
\hline 33. & Conveniencia de uso \\
\hline 34. & Reparabilidad \\
\hline 35. & Adapatibilidad \\
\hline 36. & Complejidad del dispositivo \\
\hline 37. & Complejidad de control \\
\hline 38. & Nivel de automatización \\
\hline 39. & Capacidad/Productividad \\
\hline
\end{tabular}

Fuente: Adaptado de [35].

Altshuller también extrajo de las patentes mundiales 40 principios inventivos. Éstas son las "pistas" que ayudarán a un ingeniero a encontrar una solución de alto nivel de inventiva (y patentable) al problema. Para encontrar qué principios inventivos usar, Altshuller creó la Matriz de contradicciones. La Matriz de contradicciones enumera los 39 parámetros en el eje $\mathrm{X}$ (efecto secundario indeseado) y en el eje Y (característica a mejorar). En las celdas de intersección, se enumeran los principios inventivos apropiados a utilizar para lograr una solución [35].

\section{II.5.2. LOS 40 PRINCIPIOS}

Los 40 principios inventivos que según Altshuller pueden y deben ser usados aparecen en la tabla 3.

Tabla 3. Principios de Inventiva

\begin{tabular}{|l|}
\hline 1.-Segmentación \\
\hline 2.-Extracción \\
\hline 3.-Calidad Local \\
\hline 4.-Asimetría \\
\hline 5.-Combinación \\
\hline 6.-Universalidad \\
\hline 7.-Anidamiento \\
\hline 8.-Contrapesos \\
\hline 9.-Acción contraria previa \\
\hline 10.-Acción Previa \\
\hline 11.-Estar preparado (compensar de Antemano) \\
\hline 12.-Equipotencialidad \\
\hline 13.- Inversión \\
\hline 14.-Esferoidad \\
\hline 15.-Dinamicidad \\
\hline 16.-Acción parcial o sobre acción \\
\hline 17.-Mover en una nueva dirección \\
\hline 18.-Vibración Mecánica \\
\hline 19.-Acción Periódica \\
\hline 20.-Continuar la acción útil \\
\hline 21.-Atravesar rápidamente \\
\hline 22.-Convertir daño en beneficio \\
\hline 23.-Retroalimentación \\
\hline 24.-Mediación \\
\hline 25.-Autoservicio \\
\hline 26.-Copiar \\
\hline 27.-Objeto barato/corta vida en vez de caro/muy durable \\
\hline 28.-Remplazar un sistema mecánico \\
\hline 29.-Usar construcción neumática o hidráulica \\
\hline 30.-Membranas delgadas o film flexible \\
\hline 31.-Uso de material poroso \\
\hline 32.-Cambiando el color \\
\hline 33.-Homogeneidad \\
\hline 34.-Rechazando y regenerando partes \\
\hline 35.-Transformación de estados físicos y químicos \\
\hline 36.-Transición de Fase \\
\hline 37.-Expansión térmica \\
\hline 38.-Usar oxidantes fuertes \\
\hline 39.-Medioambiente inerte \\
Fuente: [36]. \\
\hline
\end{tabular}

\section{II.5.2.1. EXPLICACIÓN DE ALGUNOS DE LOS PRINCIPIOS}

\section{Principio 1 - Segmentación}

a. Dividir un objeto en partes independientes

b. Hacer un objeto seccional

c. Aumentar el grado de la segmentación de un objeto Ejemplos:

- Los muebles seccionales, componentes de computadora modulares, reglas de madera plegables. 
- Mangueras de jardín ensamblables para formar cualquier longitud necesaria.

\section{Principio 3 - Calidad Local}

a. Transición de una estructura homogénea a una estructura heterogénea

b. Hacer que diversas partes del objeto realicen diversas funciones

c. Poner cada parte del objeto bajo condiciones más Ejemplo: favorables para su operación

- El lápiz y la goma en una sola unidad

\section{Principio 14. Esferoidad o Esfericidad}

a. Substituir las piezas lineares o las superficies planas por curvas; substituir las formas cúbicas por formas esféricas

b. Utilizar rodillos, bolas

c. Substituir el movimiento linear por el movimiento giratorio; utilizar la fuerza centrífuga

Ejemplo:

- El ratón de la computadora utiliza una construcción de bola para transferir el movimiento linear en dos ejes.

\section{Principio 4 - Asimetría}

a. Substituir una forma simétrica por una forma asimétrica.

b. Si un objeto es ya asimétrico, aumentar el grado de asimetría

Ejemplos:

- Hacer un lado de un neumático más fuerte que el otro para resistir el impacto con el contén

- Mientras que descarga la arena mojada a través de un embudo simétrico, la arena forma un arco sobre la abertura, causando flujo irregular. Un embudo de la forma asimétrica elimina el efecto de arqueamiento.

\section{Principio 40. Materiales compuestos}

a. Substituir un material homogéneo por un material Ejemplo: compuesto (composite)

- Las alas de los aviones militares se hacen de compuestos de plásticos y de fibras del carbón para alta resistencia y bajo peso.

\section{II.5.3EJEMPLOS DE CONTRADICCIONES}

En la tabla 4 se puede apreciar a manera de ejemplos las contradicciones físicas y técnicas que se pueden presentar a la hora de resolver un problema de Ingeniería.

Tabla 4. Contradicciones físicas y técnicas que se pueden presentar a la hora de resolver un problema de Ingeniería

\begin{tabular}{l|l|l|l}
\hline \multicolumn{2}{c|}{ CONTRADICCIONES TÉCNICAS (ejemplos) } & \multicolumn{2}{c}{ CONTRADICCIONES FÍSICAS (ejemplos) } \\
\hline \multicolumn{1}{c}{ Parámetro a mejorar } & Parámetro que empeora & \multicolumn{1}{c}{ Característica "A" } & Característica "no A" \\
\hline Potencia & Peso & Electroconductivo & Dieléctrico \\
\hline Complejidad & Funcionalidad & Líquido & Sólido \\
\hline Adaptabilidad & Fiabilidad & Fuerte & Suave \\
\hline Productividad & Precisión & Rápido & Lento \\
\hline Conveniencia de Uso & Facilidad fabricación & Fuerte & Débil \\
\hline
\end{tabular}

Fuente; Adaptado de [37]

\section{II.5.4. LOS PRINCIPIOS DE SEPARACIÓN.}

Casi todas las contradicciones técnicas pueden ser transformadas en una contradicción física correspondiente. Cuando se transforma una contradicción técnica a una física, se define un problema físico específico que se soluciona fácilmente con la aplicación de principios "físicos" y los efectos físicos, químicos y geométricos junto con otros fenómenos.

Cuando tratamos con una contradicción física conocida, se puede utilizar uno de los cuatro Principios de Separación para vencer este tipo de la contradicción:

- Separación de propiedades contradictorias en el tiempo.

- Separación de propiedades contradictorias en el espacio.

- Transformaciones del sistema.

- Transformaciones de fase, o transformaciones físicas y químicas de sustancias.

Cada principio de separación se debe investigar para lograr el más avanzado pensamiento.
También existen las contradicciones humanas, se deben a lo que Altshuller llamó "Inercia psicológica" que consiste en un bloqueo o resistencia a aceptar un cambio, producto de la creatividad de otros. Altshuller también diseñó herramientas para disminuir esta dificultad. Estos tres tipos de contradicciones son las que dominan cualquier sistema.

\section{II.6. TIPOS DE PROBLEMAS.}

\section{II.6.1 PROBLEMAS CON SOLUCIONES CONOCIDAS.}

Se pueden solucionar generalmente por la información encontrada en los libros, revistas técnicas, o con los expertos en el tema. Estas soluciones siguen el patrón general de solución de problemas mostrado en la figura. Aquí, el problema particular se lleva a un problema estándar de una naturaleza similar o análoga. Se conoce una solución estándar y de esa solución estándar viene una solución particular al problema (ver figura 1). 


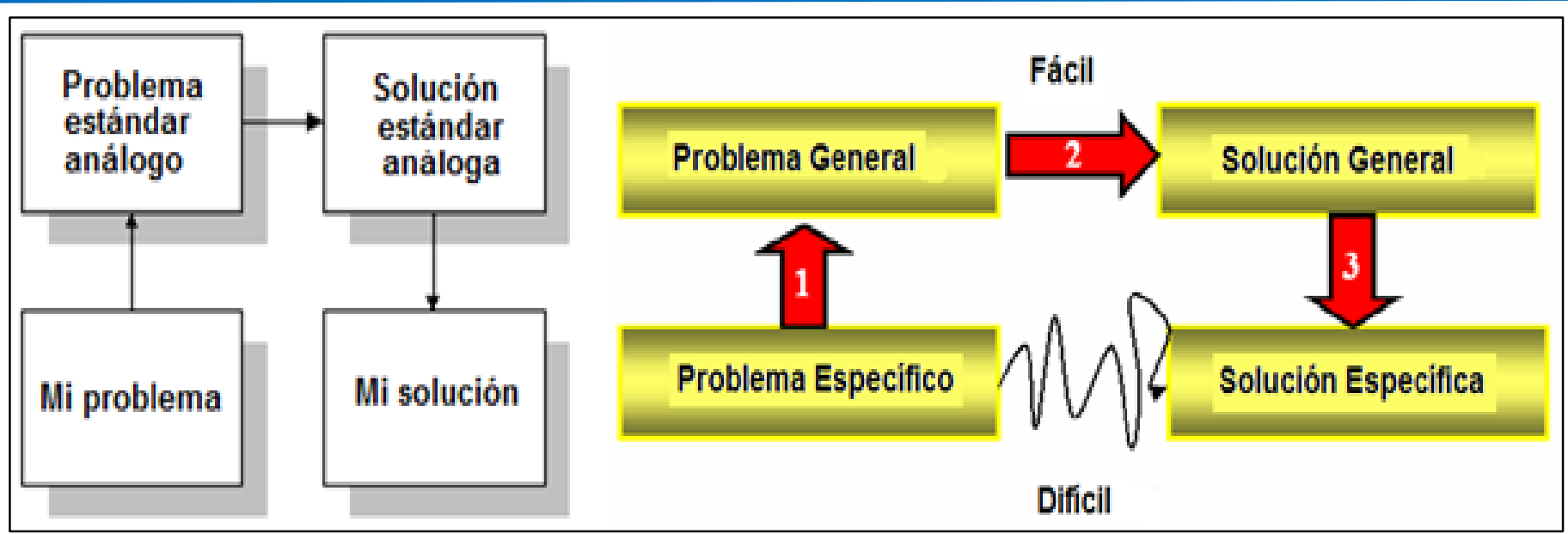

Figura 1. Proceso de plantreamiento del problema usando TRIZ.

Fuente: Los Autores, (2016).

Por ejemplo, en el diseño de una cortadora giratoria (mi problema), se requiere un motor de gran potencia pero de bajas RPM (100). Puesto que la mayoría de los motores de CA tienen altas RPM (3600 RPM), el problema estándar análogo es cómo reducir la velocidad del motor. La solución estándar análoga es una transmisión por engranajes. Entonces, se puede diseñar un reductor de engranajes con dimensiones, peso, RPM, torque, etc. apropiados para mis necesidades del corte.

\section{II.6.2. PROBLEMAS CON SOLUCIONES NO CONOCIDAS O DE INVENTIVA}

Es uno sin la solución sabida y puede contener requisitos contradictorios. En tiempos modernos, la solución de problemas de inventiva ha caído en el campo de la psicología donde se estudian los acoplamientos entre el cerebro, los métodos y la innovación. Los métodos tales como brainstorming y trial-and-error se sugieren comúnmente. Dependiendo de la complejidad del problema, el número de ensayos variará. Si la solución yace dentro del campo o experiencia de uno, tal como la ingeniería mecánica, el número de ensayos será menor. Si la solución no es visible, el inventor debe mirar más allá de su experiencia y conocimiento a nuevos campos tales como la química o la electrónica.

Entonces el número de ensayos crecerá dependiendo de cómo de bien el inventor puede dominar las herramientas psicológicas como la reunión de reflexión, la intuición, y la creatividad.

\section{II.6.2.1. INERCIA SICOLÓGICA}

Otro problema es que las herramientas psicológicas como experiencia y la intuición son difíciles de transferir a otras personas. Esto lleva a lo que se llama inercia psicológica, donde las soluciones que son consideradas están dentro de la experiencia del diseñador y las soluciones que son consideradas están dentro de la experiencia de cada cual y no se mira a tecnologías alternativas para desarrollar nuevos conceptos.
Esto es mostrado por el vector de inercia psicológica de la figura 2

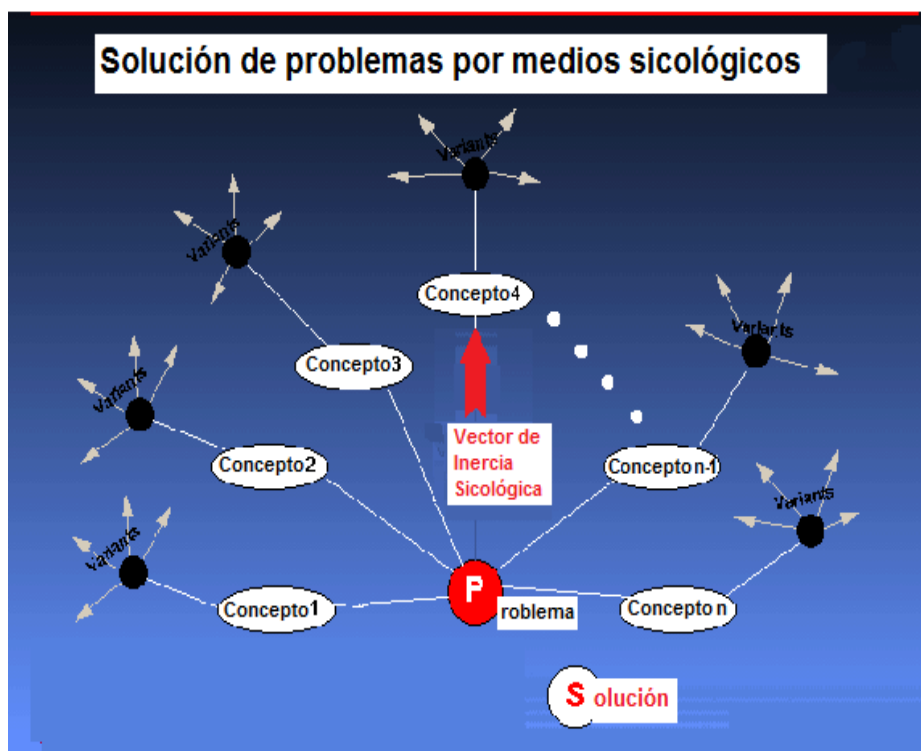

Figura 2. Efectos limitantes de la inercia sicológica.

Fuente: Adaptado de [38].

Cuando se analizan los efectos limitadores de la inercia psicológica en un mapa que cubra todas las disciplinas científicas y tecnológicas, se puede apreciar que la solución ideal puede estar fuera del campo de la experiencia del inventor. 'Esto se ve en la figura 3, donde la solución ideal sería electromecánica, pero la misma está fuera de la experiencia del ingeniero mecánico.

Si la solución de problemas fuera un proceso al azar, se esperaría que las soluciones ocurrieran aleatoriamente a través del espacio de solución. La inercia psicológica derrota la aleatoriedad y lleva a la mirada solamente a donde hay experiencia personal. 


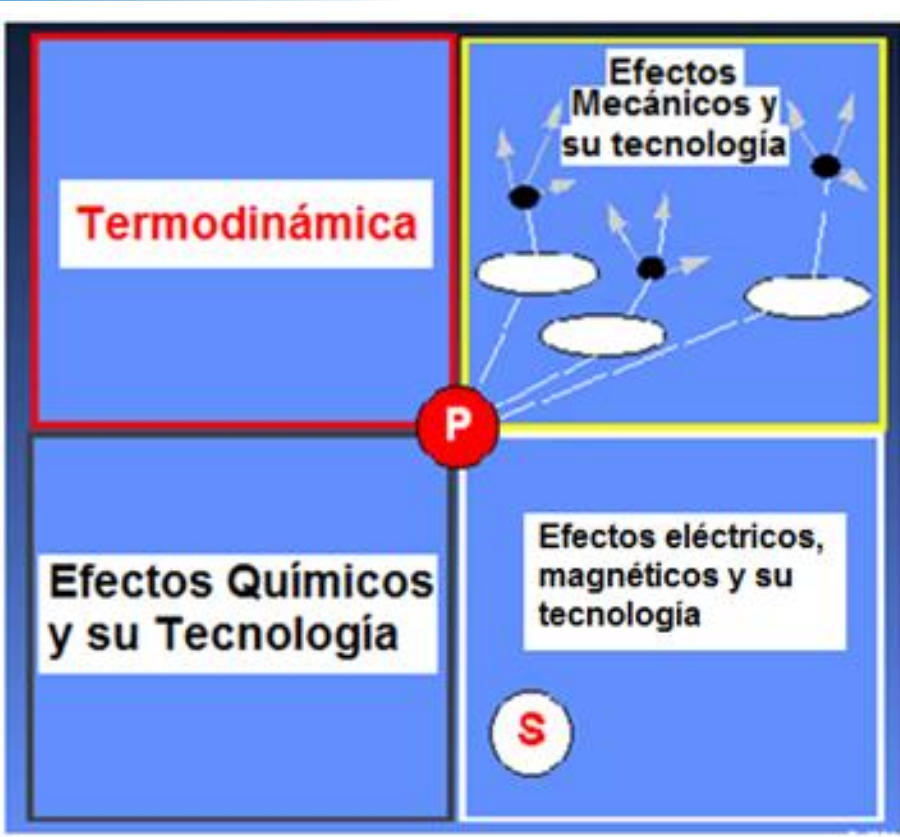

Figura 3. El problema de la inercia psicológica.

Fuente: Adaptado de [38].

\section{II.6.2.2. CONFIAR EN LA TECNOLOGÍA Y NO EN LA SICOLOGÍA}

Un mejor acercamiento, confiando no en la psicología sino en tecnología fue el desarrollado por Genrich S. Altshuller. Su curiosidad sobre la solución de problemas lo llevó a buscar métodos estándar. Al mínimo, Altshuller sentía que una teoría de la invención debía satisfacer las condiciones siguientes:

Debe ser un procedimiento sistemático paso a paso.

Debe ser una guía a través de amplios espacios de solución para dirigir los pasos a la solución ideal.

Debe ser repetible y confiable y no dependiente de las herramientas psicológicas.

Debe permitir el acceso al cuerpo del conocimiento inventivo.

Debe permitir agregar elementos al cuerpo de conocimiento inventivo.

Debe ser lo suficientemente amigable para los diseñadores siguiendo la aproximación general para la resolución de los problemas inventivos.

II.7. LA TEORÍA DE SOLUCIÓN DE LOS PROBLEMAS DE INVENTIVA. LEY DE INCREMENTO DE LA IDEALIDAD

Hay varios conceptos teóricos en la TRIZ. Uno de ellos es la ley de aumentar la "Idealidad". Esto significa que los sistemas técnicos se desarrollan hacia grados cada vez mayores de idealidad. La Idealidad es uno de los conceptos que hizo esta metodología atractiva y eficaz. Es la esencia que mueve al hombre para mejorar cualquier sistema técnico y al más bajo costo. Aumentar las funciones útiles y/o reducir las funciones perjudiciales acercan el sistema a la Idealidad. El Sistema Ideal sería aquel que no existe materialmente, pero que, aún así, realiza la función. Por lo tanto la Idealidad puede ser descrita por la expresión siguiente [39]:

$$
\mathrm{I}=\frac{\sum \mathrm{ED}}{\left(\sum \mathrm{EI}+\sum \mathrm{C}\right)}
$$

Donde:

$I=$ Sistema tecnológico Ideal.

$\sum E D=$ Sumatoria de los efectos deseados.

$\sum E I=$ Sumatoria de los efectos indeseados.

$\sum C=$ Sumatoria de los costos del sistema tecnológico. través de:

El sistema Ideal se consigue sin añadir complejidad a

- Minimizar partes

- Minimizar recursos

- Utilización de efectos químicos, físicos y geométricos

\section{II.7.1. EFECTOS ÚTILES E INÚTILES}

Los efectos útiles incluyen todos los resultados valiosos del funcionamiento del sistema.

El estado ideal es uno donde hay solamente ventajas y ningún efecto dañino. Es a este estado al que los sistemas y productos deben evolucionar. Desde el punto de vista del diseño, los ingenieros deben continuar persiguiendo mayores ventajas y reduciendo el coste de mano de obra, de materiales, de energía, y de efectos secundarios dañinos.

Normalmente, cuando la mejora de una ventaja da lugar a efectos dañinos crecientes, se hace un compromiso o trade-off, pero la ley de Idealidad conlleva a diseños para eliminar o para solucionar cualquier compromiso o contradicciones de diseño.

La evolución del reloj de resorte mecánico en el reloj electrónico del cristal de cuarzo es un ejemplo de la mudanza hacia la idealidad.

\section{II.8. EL PROCESO DE LA TRIZ PASO A PASO}

Según lo mencionado anteriormente, Altshuller sentía que una teoría aceptable de la invención debe ser bastante familiar a los inventores siguiendo el método general a la solución de problemas mostrado en la figura siguiente.

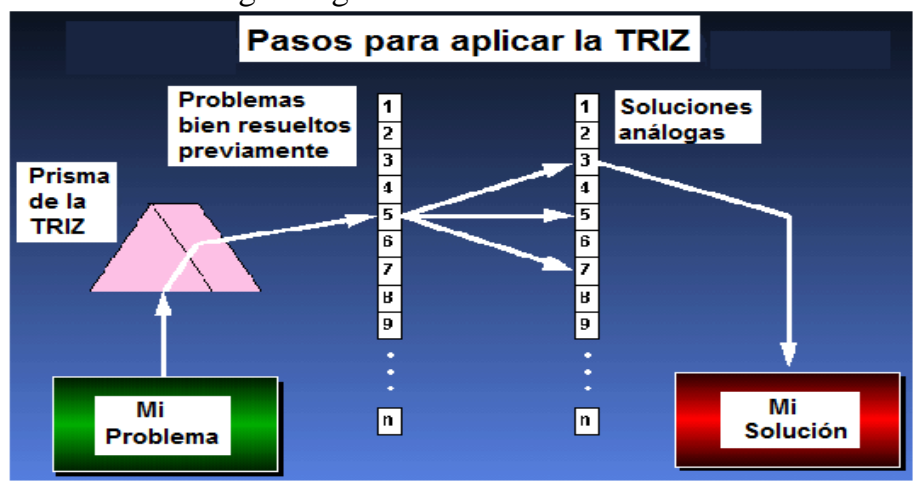

Figura 4; El proceso TRIZ paso a paso.

Fuente: Adaptado de [40]. 


\section{II.8.1. IDENTIFICAR EL PROBLEMA.}

Boris Zlotin y Alla Zusman, científicos de los principios TRIZ de la "American company Ideation" y estudiantes de Altshuller han desarrollado un "Innovative Situation Questionnaire" [41] para identificar el sistema de ingeniería que era estudiado, su ambiente de operación, requisitos de recurso, función útil primaria, efectos dañinos, y resultado ideal.

Ejemplo: Una lata de refrescos. Es un sistema de ingeniería diseñado para contener una bebida. El ambiente de operación es que las latas están apiladas para los propósitos del almacenaje. Los recursos incluyen el peso de las latas llenas, la presión interna de la lata, rigidez de la construcción de la lata. La función útil primaria es contener la bebida. Los efectos dañinos incluyen el coste de materiales y producción de la lata y la pérdida de espacio de almacenaje. El resultado ideal es una lata que pueda soportar el peso de amontonamiento a la altura humana sin daño a las latas o a la bebida en las latas

\section{II.8.2. FORMULAR EL PROBLEMA A TRAVÉS DEL PRISMA DE LA TRIZ}

Exponer el problema en forma modificada en términos de contradicciones físicas. Identificar los problemas que podrían ocurrir. ¿Podría mejorando una característica técnica para solucionar el problema causar el empeoramiento de otras características técnicas, dando por resultado el surgimiento problemas secundarios? ¿Hay los conflictos técnicos que pudieron forzar a un compromiso o trade-off?

Ejemplo: No podemos controlar la altura a la cual las latas serán apiladas. El precio de materias primas nos obliga a costos más bajos. Las paredes de la lata se deben hacer lo más finas posibles para reducir costes, pero si hacemos las paredes más finas, no pueden soportar una gran carga de amontonamiento o apilamiento. Así, la pared de la lata necesita ser más fina para bajar el coste del material y más gruesa para soportar el peso de la carga cuando están apiladas. Esta es una contradicción física. Si se puede solucionar, se alcanzará un sistema de ingeniería ideal.

\section{II.8.3. BUSCAR UN PROBLEMA BIEN SOLUCIONADO PREVIAMENTE}

Altshuller extrajo de cerca de 1.500 .000 patentes mundiales 39 características técnicas estándar que causan conflicto. Éstos como ya se expresó, se denominan los 39 parámetros de Ingeniería que pueden entrar en contradicción. Primero se encuentra el principio que necesita ser cambiado. Luego se busca el principio que es un efecto secundario indeseable. Entonces se establece el conflicto técnico estándar.

\section{II.8.4. PARÁMETROS DE INGENIERÍA}

Ejemplo. El parámetro estándar de la ingeniería que tiene que ser cambiado para hacer que la pared de la lata sea más fina es el “\#4, longitud de un objeto inmóvil” En TRIZ, estos principios estándar de la ingeniería pueden ser bastante generales. Aquí, la "longitud" puede referirse a cualquier dimensión linear tal como longitud, ancho, altura, diámetro, etc. Si se hace la pared de la lata más fina, el peso de la carga apilada disminuirá. El parámetro estándar de la ingeniería que está en conflicto es el “\#11, tensión."

El conflicto técnico estándar es: cuanto más se mejora el parámetro estándar de Ingeniería "longitud de un objeto inmóvil", más es el parámetro estándar de Ingeniería "tensión" será peor.

\section{II.8.5. BUSCAR SOLUCIONES ANÁLOGAS Y ADAPTARLAS A MI SOLUCIÓN}

Cuando hay un problema complejo en un sistema de ingeniería por lo general casi siempre hay una contradicción que puede ser técnica o física. Una contradicción técnica es un reto a vencer, cuando una mejora en un parámetro " $\mathrm{A}$ " del sistema causa un deterioro del parámetro "B". La metodología tradicional para resolver la contradicción es llegar a un compromiso entre los parámetros A y B. Mientras que la metodología de TRIZ considera la resolución de la contradicción como una mejora de la calidad influenciada por los parámetros A y B juntos.

\section{APLICACIONES DE LA TRIZ AL DISEÑO. ESTUDIO DE CASOS.}

En la figura 5 se muestra un diagrama que de manera general auxilia al usuario a usar la metodología TRIZ.

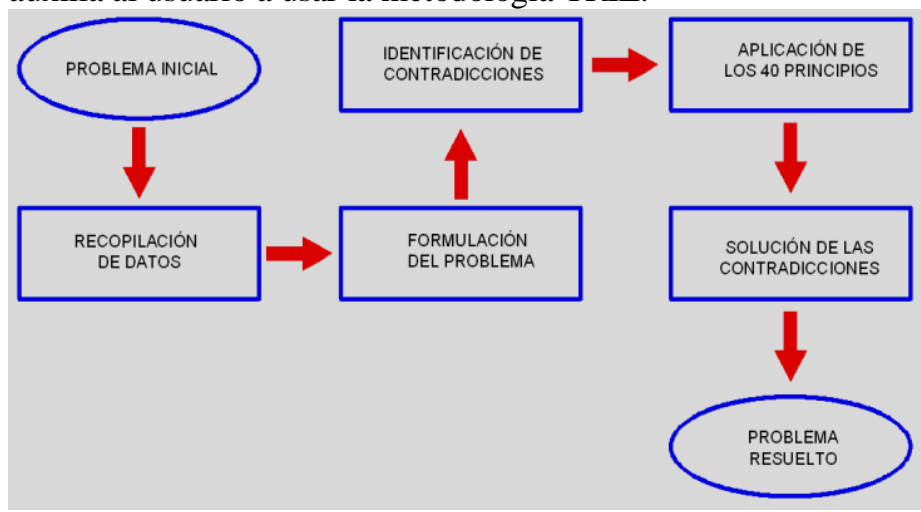

Figura 5. Esquema para resolver un problema según la metodología TRIZ.

Fuente; Adaptado de [42].

\section{III.1. ESTUDIO DE CASO 1.}

La bicicleta modelo estándar ocupa demasiado espacio para su almacenamiento, localización, transponte y manipulación.

\section{III.1.1. DECLARACIÓN DE LA CONTRADICCIÓN TÉCNICA.}

La bicicleta debe adaptarse al espacio que sea necesario, aplicando los 39 parámetros de TRIZ, se diría que se requiere mejorar la adaptabilidad o flexibilidad de la bicicleta, pero esto, a su vez, produce que la longitud del objeto móvil se vea perjudicada. En otras palabras, cuando la adaptabilidad o flexibilidad de la bicicleta mejora (parámetro 35), la longitud del objeto móvil (parámetro 3) se perjudica. Al usar la matriz de contradicciones, se muestran los principios de inventiva que se deben emplear (ver tabla 5). 
La tabla 5 ha demostrado que en realidad todos los principios tienen la misma utilidad para generar ideas innovadoras.

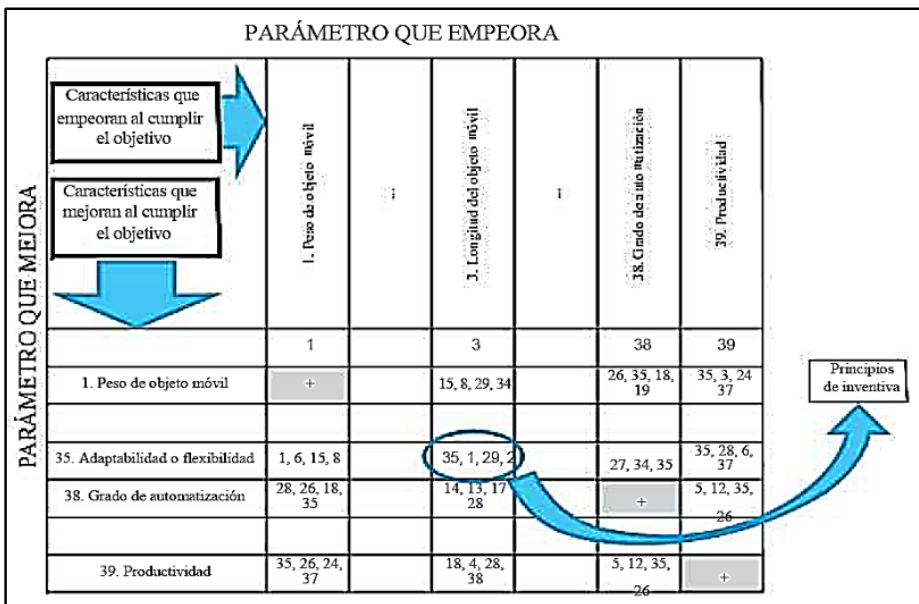

Fuente: Los Autores, (2016).

\section{III.1.2 LOS PRINCIPIOS QUE HAN DE SER EMPLEADO EN LA SOLUCIÓN DE LA CONTRADICCIÓN SON:}

No. PRINCIPIO DE INVENTIVA

35 Transformación de propiedades (cambio del estado físico)

1 Segmentación

29 Neumática e hidráulica

2 Extracción

Al emplear el principio de Segmentación, se puede hacer que la bicicleta sea un conjunto de partes, que sean fáciles de ensamblar y que la suma de las partes sea la bicicleta en sí. Cuando se piensa en cómo se podría realizar esto, se encuentra que la bicicleta, desde su estructura original, se puede juntar o reducir, es decir, hay que hacer que las partes que son uniones sólidas de la bicicleta sean movibles, para que esto permita ensamblar o desensamblar la bicicleta; así, nace lo que se conoce como "Folding bike". Es necesario aclarar que en la matriz de este ejemplo se presentan los principios de inventiva, de cada celda, en un orden supuestamente de mayor a menor relevancia. La experiencia ha demostrado que en realidad todos los principios tienen la misma utilidad para generar ideas innovadoras.

Los distintos tipos de folding bikes que existen depende de la habilidad de cada persona; lo que importa es que, en este nuevo producto se ha aplicado el principio de segmentación.

Al aplicar el principio 29 de neumática o hidráulica, se puede lograr que la estructura de la bicicleta sea de un material especial inflable, que al interactuar con un gas interno, se ponga rígido, es decir, que cuando llene de algún gas la estructura de la bicicleta, esta se ponga rígida, logrando así la estabilidad de la estructura, pero una vez usada la bicicleta se libera el gas de la estructura y solo queda la estructura maleable y las llantas; de este modo, se logra su fácil transporte o manipulación.

No siempre en obligatorio aplicar todos los principios que sugiere la matriz, puesto que estos son generales y en muchos casos no todos proceden.

\section{III.2. ESTUDIO DE CASO 2}

Diseñar una transmisión abierta de engranajes cilíndricos de dientes rectos, cuyas exigencias técnicas de diseño se muestran en la tabla 6

Tabla 6. Exigencias de la transmisión por engranajes

\begin{tabular}{|l|l|}
\hline Relación de transmisión, $\mathrm{U}_{\text {t.e. }}$ & $2,78 \mathrm{ul}$ \\
\hline Potencia de entrada, $\mathrm{P}$ & $5 \mathrm{~kW}$ \\
\hline Velocidad de entrada, $\mathrm{n}_{\text {entrada }}$ & $150 \mathrm{rpm}$ \\
\hline Eficiencia de la transmisión, $\eta_{\text {t.e. }}$ & 0,97 \\
\hline Vida útil deseada & $10000 \mathrm{hr}$ \\
\hline Material disponible & GOST 38ChA \\
\hline Posible Dureza & $45-50 \mathrm{HRC}$ \\
\hline Carga Ligera: & $120 \%$ Sobrecarga \\
\hline Tratamiento térmico & T.C.A.F \\
\hline Disposición de las ruedas & Simétricas \\
\hline \multirow{2}{*}{ El diseño requiere que la transmisión sea conservadoramente compacta. } \\
\end{tabular}

Fuente: Los autores (2016).

\section{III.2.1. DECLARACIÓN DE LA CONTRADICCIÓN TÉCNICA}

La matriz de contradicciones propone los principios de inventiva que según Altshuller deben ser empleados en la solución de la contradicción (10, 26 y 28) (ver tabla 7).

\section{III.2.2 ANÁLISIS DE CADA UNO DE LOS PRINCIPIOS DE INVENTIVA No. PRINCIPIO DE INVENTIVA}

Principio 10. Acción anticipada. (No se corresponde con la naturaleza del problema). 
Principio 26. Copiado. (No se corresponde con la naturaleza del problema)

Principio 28. Remplazar un sistema mecánico con otro sistema. (No se corresponde con la naturaleza del problema)

\section{III.2.3. SOLUCIÓN INNOVADORA:}

Luego de un estudio detallado de cada uno de los principios que sugiere la matriz, así como del resto propuesto por el profesor Altshuller, es imposible hallar un principio con una definición asociada que permita resolver la contradicción del problema específico. Se decidió entonces usar la combinación de dos principios:

- La asimetría

- $\quad$ El uso de materiales compuestos.

En la figura 6 se muestra el diente asimétrico fabricado para dar solución al problema, así como las tensiones que surgen en el mismo.
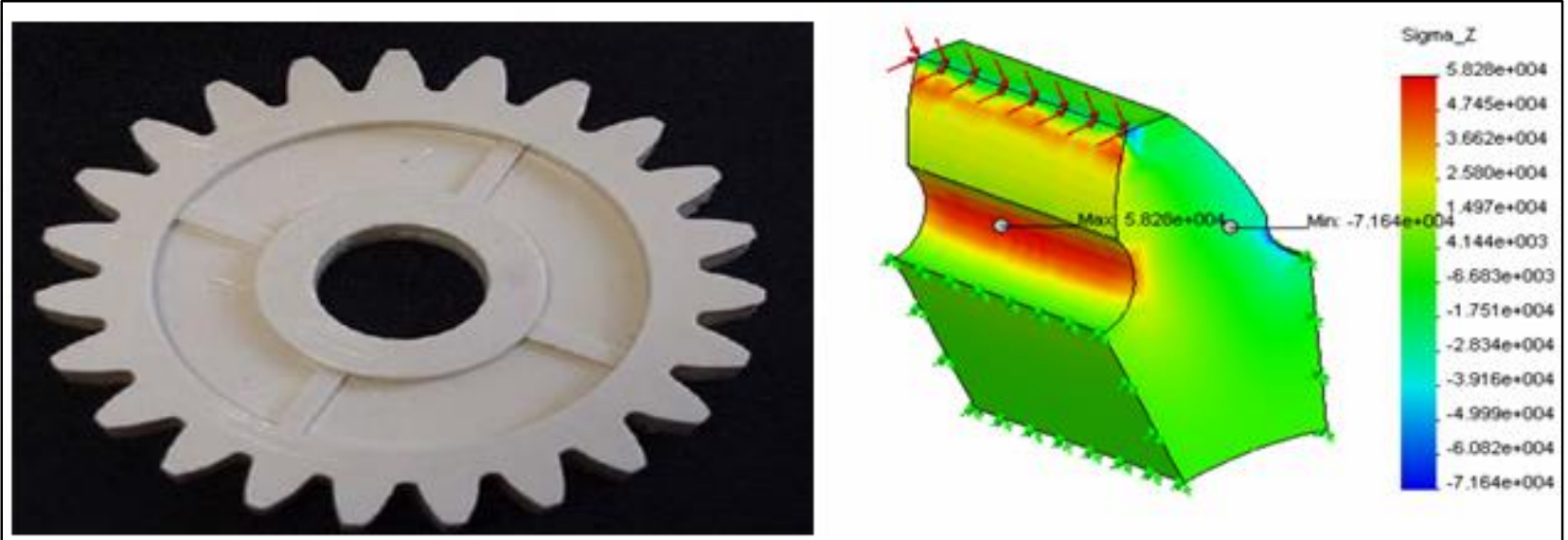

Figura 6. Diente asimétrico de Poliamida fabricado para dar solución al problema, así como las tensiones que surgen en el mismo. Fuente: Los autores, (2016).

Tabla 7. Matriz de contradicciones del estúdio de caso 2.

\begin{tabular}{|c|c|c|c|c|c|c|c|c|}
\hline \multicolumn{9}{|c|}{ PARÁMETRO O CARACTERÍSTICA QUE SE DETERIORA } \\
\hline \multirow{12}{*}{ 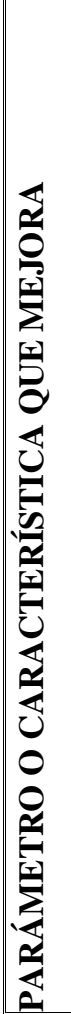 } & & & 9 & 10 & 11 & 12 & 14 & 15 \\
\hline & & & Velocidad & Fuerza & $\begin{array}{l}\text { Tensión/ } \\
\text { Presión }\end{array}$ & Forma & Resistencia & \begin{tabular}{|l|} 
uurabilidad \\
del objeto \\
móvil \\
\end{tabular} \\
\hline & 21 & Potencia & $2,15,35$ & $2,26,35,36$ & $10,22,35$ & $2,14,29,40$ & $10,26,28$ & $10,19,35,38$ \\
\hline & 22 & Pérdida de Energía & $16,35,38$ & 36,38 & & & 26 & \\
\hline & 23 & Pérdida de materia & $10,13,28,38$ & $14,15,18,40$ & $3,10,36,37$ & $3,5,29,35$ & $28,31,35,40$ & $3,18,27,28$ \\
\hline & 24 & $\begin{array}{l}\text { Pérdida de } \\
\text { información }\end{array}$ & 26,32 & & & & & 10 \\
\hline & 21 & Pérdida de tiempo & & $5,10,36,37$ & $4,36,37$ & $4,10,17,34$ & $3,18,28,29$ & $10,18,20,28$ \\
\hline & 26 & $\begin{array}{l}\text { Cantidad de sustancia } \\
\text { o de materia }\end{array}$ & $28,29,34,35$ & $3,14,35$ & $3,10,14,36$ & 14,35 & $10,14,34,35$ & $3,10,35,40$ \\
\hline & 27 & Fiabilidad & $11,21,28,35$ & $3,8,10,28$ & $10,19,24,35$ & $1,11,16,35$ & 11,28 & $2,3,25,35$ \\
\hline & 28 & $\begin{array}{l}\text { Precisión de la } \\
\text { medición }\end{array}$ & $13,24,28,32$ & 2,32 & $6,28,32$ & $6,28,32$ & $6,28,32$ & $6,28,32$ \\
\hline & 29 & $\begin{array}{l}\text { Precisión de la } \\
\text { manufactura }\end{array}$ & $10,28,32$ & $19,28,34,36$ & 3,35 & $30,32,40$ & 3,27 & $3,27,40$ \\
\hline & 39 & Productividad & & $10,15,28,36$ & $10,14,37$ & $10,14,34,40$ & $10,18,28,29$ & $2,10,18,35$ \\
\hline
\end{tabular}

Fuente: Los Autores (2016). 


\section{CONCLUSIONES}

TRIZ es una metodología de resolución de problemas basada en un acercamiento lógico y sistemático. Puede ser utilizada como un instrumento intelectual poderoso para solucionar problemas técnicos y tecnológicos, sencillos y difíciles, más rápidamente y con mejores resultados.

Los 40 Principios Inventivos son uno de los aportes más importantes del Profesor Altshuller a la ciencia y la tecnología, que a su vez son la base de la matriz de contradicciones, parte fundamental de cualquier trabajo que pretenda inventar o innovar alguna cosa. Los principios pueden utilizarse individualmente o en combinación para resolver contradicciones técnicas y finalmente solucionar el problema.

Los principios inventivos tienen su propia especificidad y requiere algunas habilidades prácticas. Son herramientas que ayudan al innovador al darle las pautas, direcciones de la innovación o creación, pero el concepto de solución concreta deberá ser el conjunto de aplicaciones de los distintos principios de TRIZ, con conocimiento personal y la creatividad humana. TRIZ ayuda a los humanos a ser creativos, pero no remplaza la creatividad humana

TRIZ es un método probado para potenciar la innovación, ayuda a afrontar problemas desde una perspectiva totalmente diferente y nueva, aportando ideas y nuevas visiones.

\section{BIBLIOGRAFÍA}

[1]. Wong, K.C., K.Z. Woo, and K.H. Woo, Ishikawa Diagram, in Quality Improvement in Behavioral Health. 2016, Springer. p. 119132.

[2]. Kogure, M., Some basic problems of quality assurance in service industries «. Total Quality Management, 1992. 3(1): p. 918.

[3]. Akao, Y., Quality function deployment. 2004.

[4]. Kano, N., Upsizing the organization by attractive quality creation, in Total Quality Management. 1995, Springer. p. 60-72.

[5]. Imai, M., Gemba Kaizen: A commonsense approach to a continuous improvement strategy. 2012: McGraw Hill Professional.

[6]. Franceschini, F., Advanced quality function deployment. 2016: CRC Press.

[7]. Marghescu, D., „Multidimensional Data Visualization Techniques for Financial Performance Data: A Review”. Turku Centre for Computer Science, 2007.

[8]. Ilevbare, I.M., D. Probert, and R. Phaal, A review of TRIZ, and its benefits and challenges in practice. Technovation, 2013. 33(2): p. 30-37.

[9]. Wang, H.C., H.P. Lin, and J. Yang. History, Current Situation and Development Prospect of the TRIZ. in Advanced Materials Research. 2013. Trans Tech Publ.

[10]. Vicentin, B.M., Metodologias de Inovação: Soluçães da TRIZ aplicadas em um ambiente design thinking. 2016.
[11]. Ciarlini, J.R., Brainfood, dude!: Manual criativo e ilustrado de brainstorming para comunicadores organizacionais. 2015.

[12]. Childs, P. and B. Garvey, Using morphological analysis to tackle uncertainty at the design phase for a safety critical application. Propulsion and Power Research, 2015. 4(1): p. 1-8.

[13]. Miles, L.D., Techniques of value analysis and engineering. 2015: Miles Value Foundation.

[14]. Ramesh, B., U. Baul, and V. Srinivasan, An approach to bolster up the logistic link of supply chain in cement industries through value engineering techniques. Indian Concrete Journal, 2016: p. 79.

[15]. Robertsingh, G., M. Sivapragash, and R. Anish, Improving Mechanical Properties of Mg Alloy by Optimizing the Composition Elements using Grey based Taguchi Method. Int. J. Applied Engineering Research, 2015. 10(8): p. 6428-6432.

[16]. Shariatmadari, A., et al., The cause and effect analysis of the dryness of Urmia Lake using a combination of fishbone diagram and analytic hierarchy process. Transylvanian Review, 2016. 24(9).

[17]. Abed, S., A.H.M. Davoudi, and D. Hoseinzadeh, The effect of synectics pattern on increasing the level of problem solving and critical thinking skills in students of Alborz province. 2015.

[18]. Zankovsky, A. and C. von der Heiden, Leadership in a Changing World, in Leadership with Synercube. 2016, Springer. p. $1-17$.

[19]. Matchett, E., Control of thought in creative work. Chartered Mechanical Engineer, 1968. 14(4): p. 163-166.

[20]. Taylor, S. and K. Littleton, Contemporary identities of creativity and creative work. 2016: Routledge.

[21]. Berger, A. and D. Sijmons, Systemic Design Can Change the World: Utopia's Practical Cousin. 2009: Sun.

[22]. Hussaini, S.W. and T. Vinnakota. Application of systemic design thinking for program management. in TENCON 2015-2015 IEEE Region 10 Conference. 2015. IEEE.

[23]. de Godoy Daiha, K., et al., Are lipases still important biocatalysts? A study of scientific publications and patents for technological forecasting. PloS one, 2015. 10(6): p. e0131624.

[24]. Hatchuel, A. and B. Weil. A new approach of innovative Design: an introduction to CK theory. in DS 31: Proceedings of ICED 03, the 14th International Conference on Engineering Design, Stockholm. 2003.

[25]. Basalla, G., The evolution of technology. 1988: Cambridge University Press.

[26]. Cohen, L., Quality function deployment: how to make QFD work for you. 1995: Prentice Hall.

[27]. Wang, J., et al. A knowledge-based system for fuzzy ECG interpretation. in First International Conference on Intelligent Systems Engineering, 1992 (Conf. Publ. No. 360). 1992. IET. 
[28]. Toffler, A. and T. Alvin, The third wave. 1981: Bantam books New York.

[29]. Pande, P.S., R.P. Neuman, and R.R. Cavanagh, The six sigma way. 2000: McGraw-Hill.

[30]. Trammell, S.R. and B.J. Davis. Using a modified HAZOP/FMEA methodology for assessing system risk. in Engineering Management for Applied Technology, 2001. EMAT 2001. Proceedings. 2nd International Workshop on. 2001. IEEE.

[31]. Constance, J., DFMA: learning to design for manufacture and assembly. Mechanical Engineering-CIME, 1992. 114(5): p. 7075 .

[32]. Clausing, D., Basic Concurrent Engineering. 1998: ASME press.

[33]. Goldratt, E.M., A meta na prática. 2006: NBL Editora.

[34]. Hammer, M. and J. Champy, Reengineering the Corporation: Manifesto for Business Revolution, A. 2009: Zondervan.

[35]. Webb, A., TRIZ: an inventive approach to invention. Engineering management journal, 2002. 12(3): p. 117-124.

[36]. Dourson, S., The 40 inventive principles of TRIZ applied to finance. the TRIZ journal, 2004. 1: p. 1-23.

[37]. Mann, D., et al., Matrix 2003: Updating the TRIZ contradiction matrix. 2003: CREAX press.

[38]. Zhang, J., K.H. Chai, and K.C. Tan, Applying TRIZ to service conceptual design: an exploratory study. Creativity and Innovation Management, 2005. 14(1): p. 34-42.

[39]. Mishra, U., Introduction to the Concept of Ideality in TRIZ. Available at SSRN 2273178, 2013.

[40]. Mann, D., An introduction to TRIZ: The theory of inventive problem solving. Creativity and Innovation Management, 2001. 10(2): p. 123-125.

[41]. Terninko, J., A. Zusman, and B. Zlotin, Step-by-step TRIZ: Creating innovative solution concepts. 1996: Responsible Management Incorporated.

[42]. Savransky, S.D., Engineering of creativity: Introduction to TRIZ methodology of inventive problem solving. 2000: CRC Press. 